it records when exposed to a standard'source. It is possible to make such a source terrestrial. Wilsing employed as his standard an electric oven, constructed as a black body, against which he calibrated a glow lamp, which in turn was compared with the stellar spectrum. His work was visual. The method requires the subsequent elimination of instrumental and atmospheric losses. The latter vary rapidly and greatly. Accordingly Wilsing's measures show a rather wide dispersion, and there are some improbable results for individual stars. Besides, the general scale seems low, so that systematic error has not been avoided. But these are minor criticisms by which one tries to learn the lesson of the difficulties. The whole work, including that of his collaborators Scheiner and Münch, is a monument of skill and care.

To vary the method as Wilsing's experience suggests, I have photographed the spectra upon panchromatic plates sensitive up to Frauenhofer's (B), and in place of the terrestrial source as standard $I$ have made comparison with a similar spectrum of Polaris, taken first and last on each plate, to guard so far as possible against atmospheric vagaries. Apart from altitude effect, it is assumed that both stars are equally affected by losses, so that there is no allowance to be made in the relative measures. We have then before us an interesting problem of interpreting the photographic deposit in terms of the active light. Different times of exposure and different wave-lengths enter with the changing sensitivity of the plate. It is in fact the basic problem of sensitometry. One might say that little can be said of it, except that it is enormously complicated. What the star contributes is merely a 'latent image' that cannot be examined, and the outcome depends on the chemicals used, their handling and temperature. The material employed is none too uniform. It is altogether a region that has hitherto been avoided, and that one would rather avoid if the gain were not so manifest of reducing it, even partially, to order. Besides, what is the alternative? There are exactly the same obscurities in using the eye, but it gives no permanent record that one can experiment on and cross-examine. Two other detectors of the same order of sensibility are offered-the selenium detector and the photo-electric cell. Whether photography may be more or less obscure than these, in a metric sense, it is certain we are more familiar with its troubles, and are in any case bound to use it for other purposes. Hence it appears to me, the sooner the discussion of the photographic deposit is reduced to a standard for scientific purposes, the better.

My own effort has been confined to reducing the complexity by defining the circumstances. If the temperature, chemicals and handling are kept the same, and if the photographic constants $p$ and $\gamma$ are separately determined in the laboratory for each batch of plates and for each wave-length used, then it would appear that the blackening of the plate can only depend on the intensity of the light and the time of exposure, or alternatively, the intensity is a function of the blackening and the time. The last two are measurable, so that to derive the former requires merely the establishment of a calibration curve in the laboratory as indicated above. Departures must be treated as accidental anomalies. It can be shown that a manageable theory may be built on this basis, applying at any rate to the case when the desideratum is the shape of the radiation curve, leading to an easy equation between the relative temperatures of two sources, as indicated from any wave-lengths at which their spectra are compared.

It will be noted that this method fails to provide an absolute standard temperature unit, as basis. This, however, may be supplied by taking the temperature of a 'Go' star the same as that of the sun; but in fact when a great many stars are used, the indeterminateness is distributed over them all, and it is found that a coherent treatment permits of very little latitude, without thrusting very improbable figures upon one or other of the extremities.

A parallel treatment of the area of the radiation curve, giving the total bolometric emission of the star, should not present any insuperable difficulty. We should then have by direct determination the two fundamental elements of the star's radiation, temperature and quantity.

The measurement of the intensity of individual lines in the spectrum promises at least as much interest as a treatment of the whole. But in writing this summary directed to a special object, I have omitted many points which mean a great deal to those conversant with astronomical literature. If we want to pass from the 'magnitude' of a star to its radiative emission, we require to know the magnitude of the sun and the distance of the star. A few decades ago the number of stellar distances known was so insignificant that no general treatment was possible. It was the want of this knowledge that really accounts for some of the deficiencies of which I have spoken above. But that is now past. Some thousands are now determined, and their number is still rapidly increasing. Of stellar masses we still have only a very few determined directly; but a well-credited theory permits us to calculate these too, even for individual stars. These are necessary adjuncts if astronomy is to unite itself with physics, and the astronomer may be relied upon not to fail in supplying them.

\title{
The Active Principles of the Pituitary Gland.
}

SINCE the discovery by Oliver and Schafer of the D blood-pressure raising or pressor effect of extracts of the posterior lobe of the pituitary gland, a number of other phenomena caused by injections of this material have been described; thus although in mammals the blood-pressure is raised, in birds it is reduced; in the former the extract also stimulates the uterus to contract, and produces an increased flow of milk and an increased secretion of urine, under certain conditions, but under others the urinary flow is markedly diminished. In frogs the extract stimulates the pigment-carrying cells or melanophores to dilate, producing thereby a darkening of the skin of the animal. It may be noted that these active principles are all obtained from the non-glandular posterior lobe of the organ, which is composed chiefly of neuroglial

$$
\text { NO. 295I, VOL. I I 7] }
$$


tissue, although it has a partial investment of glandular cells from the pars intermedia. The glandular anterior lobe, which is essential for normal growth, has not yet yielded for certainty an active extract to the labours of the biochemist.

Are these various active principles, designated in the present state of our knowledge by an adjective descriptive of the effect produced, all different, or is there only one, or possibly only two or three? Subject to the isolation of one (or more) in a chemically pure condition, we can only attempt to reach an answer to this question by consideration of the indirect evidence which is available. Before reviewing some recent work on this subject, attention may be directed to one or two points. It is generally held at the present time that the diuresis observed after injection of a posterior lobe extract intravenously into an anæsthetised animal is caused by the rise of blood-pressure simultaneously produced, with perhaps a dilatation of the kidney blood-vessels, and that the galactogogue effect is due to the same principle stimulating the smooth muscle fibres in the walls of the ducts of the mammary gland, and so leading to an expression of the milk already secreted; in neither case is it considered that the extract stimulates the kidney or mammary gland cells to increased activity. It appears almost certain that some of the effects must be pharmacological rather than physiological ; the usefulness of a principle which will produce a flow of milk and an expulsion of a fœetus from the uterus to a fish or frog is obscure.

Evidence as to the identity or otherwise of the active principles has been sought by an examination of the glands of different species for the presence of the various activities associated with gland extracts. According to a table given by L. T. Hogben and G. R. de Beer (Quart. Journ. Exper. Physiol., r925, vol. I5, p. I63), extracts of the pituitaries of elasmobranch and teleost fish, amphibia, reptiles, birds and mammals produce all the effects obtainable from such extracts, except that there is some doubt as to the presence of a pressor and diuretic principle in the gland of the skate. The authors have made quantitative comparisons of the contents of the skate's and cod's glands in the pressor and oxytocic (uterine-stimulating) principles, and have found that the latter contains twenty to thirty times as much oxytocic activity as the former, tested on the virgin guinea-pig's uterus, whilst the pressor activity of the cod's gland is at least five times more than the skate's, tested on the spinal cat. Unfortunately, the doses of skate's gland extract used failed to produce any rise of bloodpressure ; it is possible that with larger doses some rise might have been obtained, seeing the great difference in their relative oxytocic activities. Hence the experiments are inconclusive, but afford no evidence that the two principles are separate chemical compounds.

Since the principles are so widely distributed throughout the animal kingdom, the differentiation between them must be made, if at all, by other means. Is there any difference in their distribution in the gland of a single species? That is, can differences be detected between their concentrations in the pars nervosa and in the pars intermedia, both of which structures are included in the posterior lobe? Hogben and de Beer have also investigated this question and find that in the pituitary gland of the ox the pars intermedia, although containing roughly only one-fifth of the amount of the principles in the pars nervosa, yet consistently contains more of the oxytocic principle than of the pressor, although usually the difference is not great. The authors favour the view that these two principles are probably different chemical substances.

Further light is shed on the problem by greater knowledge of the chemical properties of the active substances, and of their specific or non-specific nature. Thus Hogben (ibid. p. I55), investigating the depressor effect of injections of extracts of the posterior lobe in birds, finds that other tissue extracts fail to give this response; moreover, the chemical properties of the active principle are, so far as investigated, the same as those of the other principles and different from those of a non-specific depressor such as histamine. The compound is localised mainly in the pars nervosa.

The principle influencing the secretion of the urine has been investigated in some detail by N. S. Craig (ibid. p. II9). The diuretic action of posterior lobe extracts was the first to be discovered, and it was only later found that in the disease diabetes insipidus, characterised by great thirst and the passage of large volumes of urine, subcutaneous injections abolished temporarily both the thirst and the polyuria, thus exerting an antidiuretic effect. It appears, therefore, that one of the functions of the posterior lobe of the gland is to maintain the concentration of the urine; if this function is deficient, polyuria and thirst result. Craig finds that, injected subcutaneously into unanæsthetised cats, the extract delays the diuresis produced by giving water or saline solution by mouth, but has much less effect on that following the exhibition of a solution of urea. No evidence of a delayed absorption of the fluid from the gastro-intestinal tract was obtained, so that it must be retained in the body after absorption; certain observations on the hæmoglobin percentage in human blood after the administration of fluid by mouth and the injection of pituitary extract confirm this result. The author inclines to the view that the principle exerts its influence by an action on the cells of the kidney.

It is usually stated that extracts of the posterior lobe stimulate all forms of smooth muscle. A. D. Macdonald (ibid.p. r9I), as the result of an investigation of their action uponisolated strips of smooth muscle from the mammalian ileum, disagrees with this statement. He finds that not all commercial extracts stimulate this preparation, that the stimulating substance can be preferentially extracted from laboratory-desiccated posterior lobe by alcohol, which extracts the nonspecific depressor compound, and also to a certain extent by ether, which extracts neither the pressor nor the oxytocic principles, that it is present in other tissues, and hence is not a specific principle of the pituitary gland, and that it is not destroyed by boiling in dilute alkali, which treatment deleteriously affects both the pressor and oxytocic activities. It is probable, therefore, that the stimulating substance is histamine, which occurs in most tissue extracts, and that it is not quite completely removed from desiccated posterior lobe preparations by alcoholic extraction; although a preparation thus treated may show no depressor effect on injecting a second dose into a mammal, yet sufficien $_{t}$

NO. 295I, VOL. I I 7] 
histamine may have been left behind to stimulate the smooth muscle of the intestine, since the latter is about one hundred times more sensitive to histamine than it is to pituitary extract. It is possible, from some experiments on the heart of the dogfish, that small quantities of choline are also present in most posterior lobe extracts. Hence it must be concluded that any gut-stimulant action which pituitary extracts possess is very largely or entirely due to the presence therein of the non-specific stimulants histamine and choline.

Finally, reference must be made to the attempted chemical separation of the active principles. As W. Schlapp (ibid. p. 327) points out, the identical behaviour of the different principles through a large number of chemical manipulations is not sufficient evidence of their chemical identity, more especially as the non-specific histamine apparently accompanies them in many cases. On the other hand, a separation of two by a single chemical treatment would argue very strongly in favour of their non-identity. The author finds that the pressor, oxytocic and melanophore principles are all destroyed at the same rate by boiling in dilute acid, but that the pressor and melanophore activities are not extracted by butyl alcohol, whereas the oxytocic largely passes into this solvent; furthermore, lead sulphide adsorbs almost all the pressor and melanophore material present in a solution, but only about half of the oxytocic. He concludes that the pressor (and melanophore) and oxytocic activities are mediated by two separate and distinct substances, thus agreeing with Hogben and de Beer, who.by a different method reached a similar conclusion. Now the melanophore principle appears to exist in greater concentration in the pars intermedia than in the pars nervosa, thus resembling the oxytocic but differing from the pressor principle. Hence it appears probable that these three activities of posterior lobe extracts are due to the presence in them of three different chemical compounds. Their relationship to the avine depressor and antidiuretic principles requires further research for its elucidation; whilst Macdonald's results throw doubt on the specificity of the galactogogue effect. The conclusions reached from a consideration of the work mentioned above agree in general with those which may be drawn from the experiments of other investigators.

\section{Vital Statistics of Scientific Academies. ${ }^{1}$}

A COMPARISON of the life statistics of such A bodies as the Royal Society of London and the scientific academies in other countries is interesting, because the efficiency and influence of these institutions depend to some extent on the average age of their membership. Unless they contain a sufficient sprinkling of the younger generation and elections are not delayed until a man has done his best work, the institution will cease to command confidence and be hampered in the fulfilment of the object for which it was founded. We welcome the publication of the statistics collected by Prof. Raymond Pearl, and published by the National Academy of Sciences of Washington, because it allows us - so far as the limited material permits - to form a judgment of the effects of the conditions of election, which differ materially from those applying to the Royal Society. The latter, as is well known, has elected since 1848 fifteen members every year, while the leading scientific body in the United States has followed the practice of Continental academies and limited its total membership.

A statistical inquiry was first made in England by General Strachey (Proc. Roy. Soc., vol. 51, I892) in the latter part of the last century, primarily for the purpose of forecasting the ultimate membership of the Royal Society, which had fallen from 768 in 1848 to 427 in I89 I (not including 36 fellows elected under a special rule). There were then still 26 members alive who were fellows in 1848 , when the statutes were changed.

In the United States the problem is reversed, the question being, what the number of elections per year should be so that the Academy might settle down at the maximum membership fixed by charter in 1863 at $25^{\circ}$.

The National Academy started with a membership of 48 , and, anxious no doubt to set a high standard, it was slow in making any substantial additions. During the first twenty years of its existence ninety-five new

1 Vital Statistics of the National Academy of Sciences: By Raymond Pearl. Proceedings of the National Academv of Sciences (vol. Ir, No. 12, 1925). members-- that is, less than five per year-were elected, and the number of elections diminished still further in the second period of twenty years, when the annual average of new members fell to a fraction more than three. Since $19 \circ 5$ the Academy increased more rapidly " under pressure of an arbitrary rule which virtually forced the election of I5 per annum." Reference to this rule occurs twice on the same page in Prof. Pearl's publication, but its exact terms are not stated.

The average age of members of the National Academy on May I, 1925, was practically identical with that which $I$ found for fellows of the Royal Society at the beginning of 1923 , being 60.7 years in one case and 60.9 years in the other. But there is a great contrast in the admission of men below middle age. During the sixty years that have elapsed since the first nominations under the charter, Washington has elected only one man below the age of thirty, while the Royal Society has elected twenty-nine in the course of seventy-five years. The same tendency is shown in elections below the age of forty years. The percentage of elections under that age was $33^{\circ} 9$ at the Royal Society and only 19.9 at Washington. The further fact emerges that the election of younger men to the National Academy has very markedly and steadily decreased since its foundation. On this point Prof. Pearl makes the following startling remarks :-

\footnotetext{
" It is easy to attribute the changing habits of the Academy relative to the election of young men to a growing conservatism of that body itself. That this is the sole cause I doubt. It is at least possible, and I incline personally to think it probable, that the increasing organization, standardization, mechanization, and constant striving for efficient mediocrity in all our academic life, which every thoughtful person has seen going on during the past 30 years, and which some have deplored and vainly endeavoured to stop, is showing as one of its most dreadful effects the curbing and fettering of the progress of the really
} brilliant student." NO. 295 I, vOL. II 7$]$ 